\title{
Effect of Axonal Length on Direct Neuromuscular Neurotization: An Experimental Study
}

\author{
Musa Kemal KELES ${ }^{1}$, Andac AYKAN² \\ ${ }^{1}$ Diskapi Yildirim Beyazit Training and Research Hospital, Department of Plastic, Reconstructive and Aesthetic Surgery, Ankara, Turkey \\ ${ }^{2}$ Yeditepe University Department of Plastic, Reconstructive and Aesthetic Surgery, Istanbul, Turkey
}

\section{ABSTRACT}

AIM: To compare the effect of long and short axonal lengths on neuromuscular neurotization (NMN).

MATERIAL and METHODS: In this study, 18 male Wistar rats were divided into two groups. In each group, the peroneal nerve and soleus muscle were dissected. In Group1, the muscle was neurotized after the peroneal nerve was dissected until bifurcation. In Group2, the nerve was transected $1 \mathrm{~cm}$ proximal from the most distal site, and the muscle was neurotized with a shorter nerve than that in Group1.

RESULTS: In Group2 (0.84), the compound muscle action potential amplitude ratio was statistically higher than that in Group1 (0.42). Upon pathological examination, the cross-sectional area was statistically larger in Group2. Acetylcholinesterase enzyme levels in Groups1 and 2 were 37.73 and 45.47, respectively.

CONCLUSION: Considering that NMN with shorter axons showed better results than that with longer axons, nerve transfers using nerves as short as possible should be preferred in clinical applications.

KEYWORDS: Axon, Experimental, Neurotization, Rat

\section{INTRODUCTION}

$\mathrm{N}$ euromuscular neurotization (NMN) involves direct implantation of a donor nerve into a denervated muscle and is used to treat chronic denervation and severe nerve injuries. Although many studies have been conducted on NMN, its exact mechanism remains unclear and the optimal technique has not been established. Numerous factors affect the results of a procedure. Various surgical techniques and exogenous factors have been studied to improve the outcome, but the clinical use of NMN remains unsatisfactory $(2,4,5,15,18,22,23,29)$.

A number of changes occur in the proximal axon, nerve cell body, and myelin sheath following axonal injury (11). The proximal nerve segment can undergo apoptosis or chromatolysis. Chromatolysis, an attempt to repair the nerve, is accompanied by an increase in the production of structural and growth cone components needed for axonal growth, i.e., tubulin, actin, and growth-associated protein $43(12,25,28)$. The location of axonal injury is critical in neuronal regeneration following a proximal nerve injury, and the consequences are more severe (10).

Thus, the objective of the current study was to compare the effect of long and short axonal lengths on NMN in a rat model of muscle denervation.

\section{MATERIAL and METHODS}

Eighteen male albino Wistar rats, weighing 275-300 g, were used. All procedures were performed in accordance with the animal research committee of our medical academia (ETiK-2014/49). Intraperitoneal ketamine, $100 \mathrm{mg} / \mathrm{kg}$, and 
intramuscular xylazine, $35 \mathrm{mg} / \mathrm{kg}$, were used for general anesthesia in all procedures. Standard microvascular instruments and a surgical microscope were used.

\section{Study Groups}

The rats were randomly divided into two groups. In both groups, the common peroneal nerve was transected from the most distal part before branching off. In Group2, an additional $1 \mathrm{~cm}$ axon was excised. All rats were euthanized on the third postoperative month, and electromyographic, biochemical, and pathological analyses were performed. Contralateral legs were used as controls for electromyography (EMG).

\section{Surgical Procedure}

The posterior gluteal region was shaved, prepared, and draped under sterile conditions. Each rat was fixed on the operating tray in the prone position. The sciatic, tibial, and common peroneal nerves were explored via a posterior incision on the right side. In Group1, the peroneal nerve was transected just before bifurcating to the deep and superficial branches. In Group2, the nerve was transected from the branching site, as

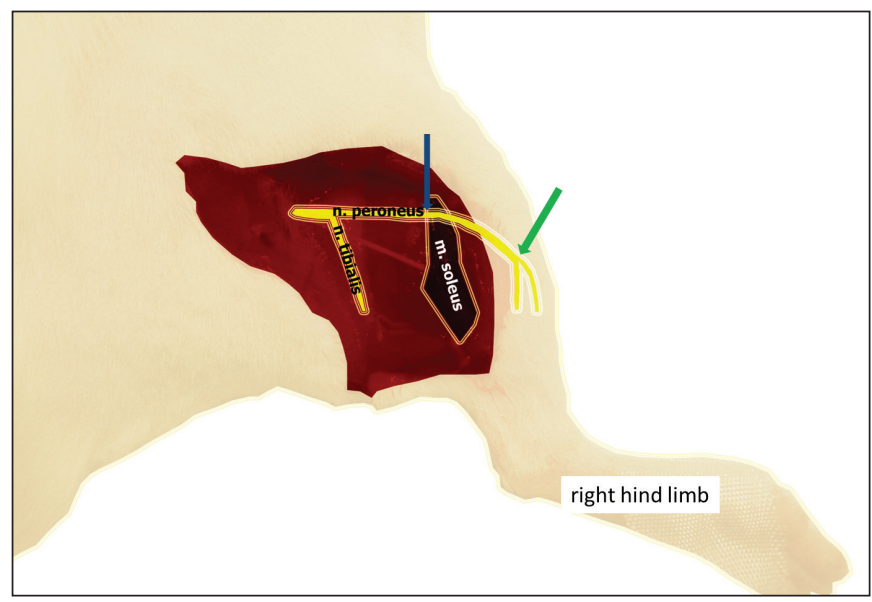

Figure 1: A schematic drawing of the experimental groups. Nerve transection was performed from the most distal part of the peroneal nerve prior to bifurcation (green arrow) in Group1. A 1 $\mathrm{cm}$ nerve segment was excised, and neuromuscular neurotization was performed (blue arrow) in Group2, following transection of the nerve from the most distal part. in Group1. Then, a $1 \mathrm{~cm}$ proximal nerve segment was excised (Figure 1).

The incision was extended to the lower leg, and the soleus muscle was explored. The branches of the tibial nerve that innervate the soleus muscle were identified and confirmed with $0.5 \mathrm{~mA}$ nerve stimulator. The muscle was then denervated, and the common peroneal nerve was inserted into the soleus muscle. The NMN was performed in the proximal one fourth of the soleus muscle in both groups (Figure 2A, B). The skin incision was closed with interrupted $5-0$ silk sutures, and the animals were returned to their cages with free access to food and water. Acetaminophen (50 mg/kg i.p.) and cefazoline (10 $\mathrm{mg} / \mathrm{kg}$ i.p.) were administered for postoperative analgesia and infection prevention.

\section{Electrophysiological Studies}

Compound muscle action potentials (CMAPs) were recorded under general anesthesia for all animals at the end of the third month. After anesthesia induction, the soleus muscle and peroneal nerve were exposed, the needle was inserted into the middle portion of the muscle, and the CMAP was recorded. The same procedure was applied to the same part of the muscle and to the contralateral legs of all animals. All investigators involved in EMG procedures were blinded to the study groups. The difference in latency was calculated by subtracting the right leg latency from the left leg latency. The amplitude ratio was calculated by dividing the right leg amplitude by the left leg amplitude.

\section{Biochemical Assays}

After the EMG, the soleus muscle was excised and divided into two pieces. The proximal half of the muscle, including the NMN site, was sent to the biochemistry laboratory. Each tissue sample was homogenized with $810 \mu \mathrm{L}$ of $0.1 \mathrm{M}$ icecold phosphate buffer saline ( $\mathrm{pH} 7.4)$. The homogenates were centrifuged at $2300 \mathrm{~g}$ for 20 mins $\left(4^{\circ} \mathrm{C}\right)$. Supernatants were removed and analyzed for protein and acetylcholinesterase (AChE) levels. The protein level in supernatant samples was measured by the Lowry method (17). The ELISA kit for rat AChE was purchased from $\mathrm{YH}$ Biosearch Laboratory (Shanghai YeHua Biological Technology Co., Ltd., Shanghai, China). ELISA assay was performed according to the manufacturer's instructions. The AChE results were divided by protein amount and given as $\mathrm{U} / \mathrm{g}$ of protein.

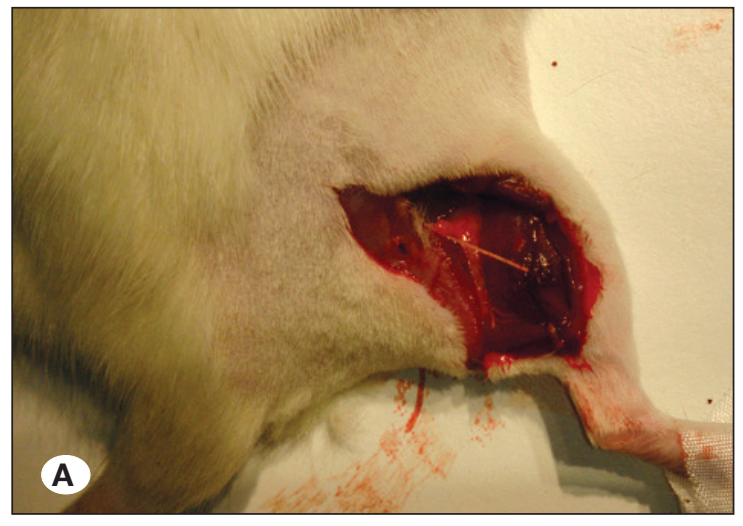

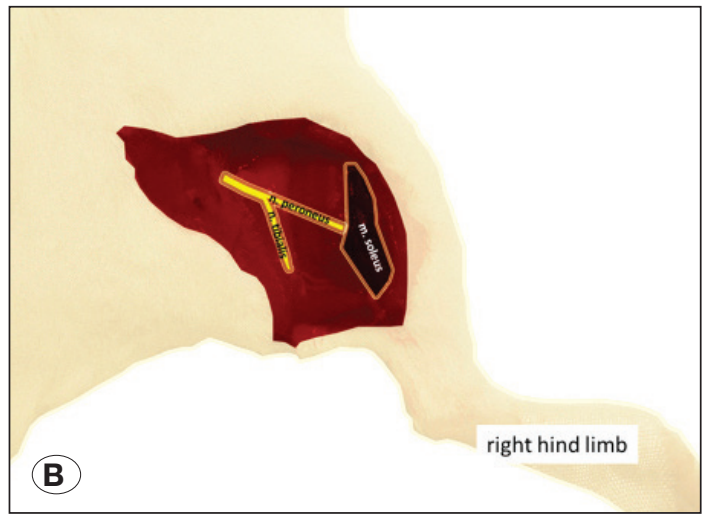

Figure 2: A depiction of the neuromuscular neurotization procedure. A) The distal stump was implanted in the soleus muscle following nerve transection. Implantation was carried out in the same part (1/4 proximal) of the muscle in both groups. B) A schematic demonstration of the surgical procedure. 


\section{Histopathological Evaluation}

The distal halves of the muscle obtained for histological studies were separately fixed in a neutral formalin solution with $10 \%$ buffer for 48 hours, then dehydrated in alcohol, and embedded in paraffin blocks. Transverse sections were cut at $4 \mu \mathrm{m}$ thickness, deparaffinized, and then stained with Masson's trichrome and hematoxylin and eosin. The tissue samples were examined with a standard light microscope. Photomicrographs were obtained at $200 \times$ magnification using an Olympus BX51 light microscope and DP71 digital camera (Olympus Co., Tokyo, Japan). For each subject, 30 muscle fascicles were randomly outlined and cross-sectional areas were quantified by pixel count using ImageJ software (version 1.50i, National Institutes of Health, Bethesda, MD, USA).

\section{Statistical Analysis}

Comparisons of electrophysiological, biochemical, and pathological results among the groups were performed using t-tests for data that were normally distributed and MannWhitney tests for data not normally distributed. Results yielding $p$ values less than 0.05 were considered statistically significant.

\section{RESULTS}

Two rats demised; one intraoperatively and the other due to an infection on day 5 postoperatively. They were replaced and the same surgical protocol was applied to the new animals. The weight of the animals was stable throughout the study.

\section{Electrophysiological studies}

Although there was no significant finding for the difference in the latency between both groups, a statistically significant difference was found for the amplitude ratio. At the end of the third month, for Group1, the mean difference in latency was $0.15 \pm 0.06$ and the mean amplitude ratio was $0.42 \pm$
0.02 . For Group2, the mean difference in latency was 0.16 \pm 0.07 , and the mean amplitude ratio was $0.84 \pm 0.05$. The difference in amplitude ratios between Groups 1 and 2 was statistically significant $(p=0.01)$. No group differences $(p>0.05)$ were observed for difference in latency (Table I).

\section{Biochemical Assays}

Although AChE levels were higher in Group1, the difference between the two groups was not statistically significant ( $p>$ 0.05). The mean AChE levels were $37.73 \pm 15.43 \mathrm{U} / \mathrm{g}$ and $45.47 \pm 14.19 \mathrm{U} / \mathrm{g}$ of protein for Groups 1 and 2, respectively (Table II).

\section{Histopathological evaluation}

For Group1, the mean cross-sectional area contained 21,027 $\pm 6,887$ pixels, whereas the mean cross-sectional area for Group2 contained $30,170 \pm 8,168$ pixels. A statistically significant group difference $(p=0.043)$ was found for pixel count (Table III).

\section{DISCUSSION}

Normally innervated muscles do not permit reimplantation by another nerve. Using NMN, the original muscle nerve can be cut to change the supplying nerve so that the inserted nerve is accepted by the denervated muscle in order to generate new motor endplates (22). Although NMN is not commonly used in nerve repair, it is sometimes the only option for some patients, meaning that the need to identify ways to improve this technique is crucial.

Numerous studies have been conducted in this regard, but the exact mechanism underlying NMN is not fully understood $(2,4,5,15,18,22,23,29)$. Several aspects of the procedure, including the source of the axon, neurotization site, depth of the neurotized axons, number of neurotized fascicles, denervation duration prior to neurotization, and

Table I: Mean Difference in Latency and Mean Amplitude Ratios of Compound Motor Action Potential in Experimental Groups at the end of the Third Month

\begin{tabular}{lccc}
\hline & Group1 & Group2 & p \\
\hline Mean difference of latencies $(\mathrm{msec})$ & $0.15 \pm 0.05$ & $0.16 \pm 0.08$ & 0.98 \\
\hline Mean amplitude ratios $(\mathrm{mV})$ & $0.42 \pm 0.10$ & $0.84 \pm 0.12$ & $\mathbf{0 . 0 1}$ \\
\hline
\end{tabular}

Table II: Mean Acetylcholinesterase Levels in Neuromuscular Junctions in Experimental Groups at the end of the Third Month

\begin{tabular}{lccc}
\hline & Group1 & Group2 & p \\
\hline Mean AchE levels (U/g protein) & $37.73 \pm 15.43$ & $45.47 \pm 14.19$ & 0.348 \\
\hline
\end{tabular}

Table III: The Mean Cross-Sectional Area was Calculated (Pixel Count) by imageJ Software. After Standard Photomicrographs were Taken, 30 Fascicles were Outlined Using Software and the Mean Area of the Fascicules was Calculated

\begin{tabular}{lccc}
\hline & Group1 (pixels) & Group2 (pixels) & p \\
\hline Mean cross-sectional area in pixels & $21027.06 \pm 6887$ & $30170.00 \pm 8168$ & $\mathbf{0 . 0 4 3}$ \\
\hline
\end{tabular}


neurotransmitter effects have been examined in previous studies $(1,3,6,8,13-16,19,21,27,30,31)$. However, the effect of axonal length on NMN outcomes has not yet been studied, and a guidance model has not been created nor established for this purpose. To address the lack of data, an animal model was designed in which the axonal length could be changed by trimming the distal stump of the peroneal nerve. Rats of similar weight were chosen to standardize the nerve length. The nerve was implanted in the soleus muscle, which was previously described and used in NMN, owing to its ideal morphological properties $(7,23)$.

The lesion level is the primary factor affecting the outcome of end-to-end nerve repair. Poor results have been attributed to proximal lesions, with evidence to support this assumption $(9,20,24,26)$. Various factors explain the difference in outcomes between proximal and distal lesions. For example, the nerve cell faces severe consequences after losing a large percentage of its volume following proximal injury. A long period is needed for the nerve to regenerate and reach its end organ with this type of injury. Another possibility is that proximal tributaries siphon off the regenerating axons, thereby decreasing the number of axons growing distally. A longer duration between injury and repair may reduce the functionality of the distal pathway and that of the end organs through scarring and atrophy. However, these mechanisms do not relate to the regeneration capacity of the peripheral nerve cell.

Metabolic activity increases in the neuron cell body after axotomy, and protein production begins to regenerate the distal stump of the axon (11). Thus, the current study hypothesized that the distance between the axon cell body and the distal axonal stump would affect regeneration and neurotization capacity via metabolic activity.

Electrophysiological, biochemical, and histological tests were performed to evaluate muscle reinnervation. EMG was carried out to facilitate a functional evaluation. An increased amplitude was observed in Group2, indicating enhanced axon sprouting from the distal stump. Other than the amplitude, a statistically significant difference was not found between the two groups. Latency is reflective of injury to the myelin sheath. A difference was not observed between the groups in terms of the myelin sheath status following the intervention in the current study.

AChE levels, a catalytic enzyme that is secreted from the postsynaptic membrane into the synaptic space, were determined to evaluate the new neuromuscular plates. AChE is mainly located at the neuromuscular junction, and its level correlates with the number of the neuromuscular junctions. In the current study, although a statistically significant difference between the groups was not found inAChE levels, they were found higher in Group2, potentially indicative of enhanced healing at the neuromuscular junction and of the presence of shorter axons. In line with this finding, the biochemical, pathological, and EMG results were more severe in Group1, while less muscular atrophy was observed in Group2.

The current study has some limitations. The number of animals included in the experiment and the study duration were limited by the ethical committee. Accordingly, only a few rodents were included, and the time available for regeneration was checked. Besides, the use of a larger animal (i.e., a monkey) is warranted as a longer nerve length would better mimic a clinical situation than a rat nerve, but this was not possible in the current study.

\section{- CONCLUSION}

Shorter axonal length was demonstrated to improve NMN healing in this rat model. To the best of our knowledge, this has not previously been achieved. When surgeons need to perform NMN (nerve transfer in congenital disorders or as a result of trauma), the use of shorter axons could yield results better than those of longer axons. Moreover, with this animal model, further research into NMN pathophysiology using different variables is warranted.

\section{- REFERENCES}

1. Askar I, Sabuncuoglu BT: Superficial or deep implantation of motor nerve after denervation: An experimental studysuperficial or deep implantation of motor nerve. Microsurgery 22(6):242-248, 2002

2. Becker M, Lassner F, Fansa $H$, Mawrin C, Pallua $\mathrm{N}$ : Refinements in nerve to muscle neurotization. Muscle Nerve 26(3):362-366, 2002

3. Brunelli G: Direct neurotization of severely damaged muscles. J Hand Surg 7(6):572-579, 1982

4. Brunelli GA: Direct neurotization of muscles by presynaptic motoneurons. J Reconstr Microsurg 17(8):631-636, 2001

5. Brunelli GA, Brunelli GR: Direct muscle neurotization. J Reconstr Microsurg 9(2):81-90; discussion 89-90, 1993

6. Chiu DT, Chen L, Spielholtz N, Beasley RW: A comparative electrophysiological study on neurotisation in rats. J Hand Surg Edinb Scotl 16(5):505-510, 1991

7. Duchen LW: An electron microscopic comparison of motor end-plates of slow and fast skeletal muscle fibres of the mouse. J Neurol Sci 14(1):37-45, 1971

8. Fukuda $A$, Hirata $H$, Akeda $K$, et al: Enhanced reinnervation after neurotization with Schwann cell transplantation. Muscle Nerve 31(2):229-234, 2005

9. Grinsell D, Keating CP: Peripheral nerve reconstruction after injury: A review of clinical and experimental therapies. BioMed Res Int 2014:698256, 2014

10. Gu Y, Spasic Z, Wu W: The effects of remaining axons on motoneuron survival and NOS expression following axotomy in the adult rat. Dev Neurosci 19(3):255-259, 1997

11. Hanz S, Fainzilber M: Retrograde signaling in injured nerve -the axon reaction revisited. J Neurochem 99(1):13-19, 2006

12. Hoffman PN, Lasek RJ: Axonal transport of the cytoskeleton in regenerating motor neurons: Constancy and change. Brain Res 202(2):317-333, 1980

13. Keilhoff $G$, Fansa $H$ : Successful intramuscular neurotization is dependent on the denervation period. A histomorphological study of the gracilis muscle in rats. Muscle Nerve 31(2):221228, 2005 
14. Kermer C, Millesi W, Paternostro T, Nuhr M: Muscle-nervemuscle neurotization of the orbicularis oris muscle. J CranioMaxillo-facSurg Off PublEurAssoc Cranio-Maxillo-fac Surg 29(5):302-306, 2001

15. Kermer C, Millesi W, Paternostro T, Nuhr M, Sabbas A: Experimental study of muscle-nerve-muscle neurotization. $J$ Reconstr Microsurg 16(7):569-572, 2000

16. Leong J, Hayes A, Austin L, Morrison W: Muscle protection following motor nerve repair in combination with leukemia inhibitory factor. J Hand Surg 24(1):37-45, 1999

17. Lowry $\mathrm{OH}$, Rosebrough NJ, Farr AL, Randall RJ: Protein measurement with the Folin phenol reagent. J Biol Chem 193(1):265-275, 1951

18. Meals RA, Nelissen RG: The origin and meaning of "neurotization." J Hand Surg 20(1):144-146, 1995

19. Menderes A, Yilmaz M, Vayvada H, Ozer E, Barutçu A: Effects of nerve growth factor on the neurotization of denervated muscles. Ann Plast Surg 48(4):415-422, 2002

20. Menorca RMG, Fussell TS, Elfar JC: Nerve physiology: Mechanisms of injury and recovery. Hand Clin 29(3):317-330, 2013

21. Papakonstantinou KC, Terzis JK, Kamin E, Luka J: Early effect of gene therapy on a direct muscle neurotization model. $J$ Reconstr Microsurg 21(6):383-389, 2005

22. Park DM, Shon SK, Kim YJ: Direct muscle neurotization in rat soleus muscle. J Reconstr Microsurg 16(5):379-383, 2000

23. Payne SH, Brushart TM: Neurotization of the rat soleus muscle: A quantitative analysis of reinnervation. J Hand Surg 22(4):640-643, 1997
24. Ruijs ACJ, Jaquet J-B, Kalmijn S, Giele H, Hovius SER: Median and ulnar nerve injuries. A meta-analysis of predictors of motor and sensory recovery after modern microsurgical nerve repair. Plast Reconstr Surg 116(2):484-494; discussion 495-496, 2005

25. Skene JH, Jacobson RD, Snipes GJ, McGuire CB, Norden JJ, Freeman JA: A protein induced during nerve growth (GAP-43) is a major component of growth-cone membranes. Science 233(4765):783-786, 1986

26. Sunderland S: The anatomy and physiology of nerve injury. Muscle Nerve 13(9):771-784, 1990

27. Swanson AN, Wolfe SW, Khazzam M, Feinberg J, Ehteshami J, Doty S: Comparison of neurotization versus nerve repair in an animal model of chronically denervated muscle. J Hand Surg 33(7):1093-1099, 2008

28. Tetzlaff W, Bisby MA, Kreutzberg GW: Changes in cytoskeletal proteins in the rat facial nucleus following axotomy. J Neurosci Off J Soc Neurosci 8(9):3181-3189, 1988

29. Ueda K, Harii K: Prevention of denervation atrophy by nerve implantation. J Reconstr Microsurg 20(7):545-553, 2004

30. Urbanchek MG, Ganz DE, Aydin MA, van der Meulen JH, Kuzon WM: Muscle-nerve-muscle neurotization for the reinnervation of denervated somatic muscle. Neurol Res 26(4):388-394, 2004

31. Zheng H, Zhou S, Chen S, Li Z, Cuan Y: An experimental comparison of different kinds of laryngeal muscle reinnervation. Otolaryngol- Head Neck Surg Off J Am Acad Otolaryngol-Head Neck Surg 119(5):540-547, 1998 